\title{
Las tecnologías de la información y su relación con la escritura
}

\author{
Information technologies and their relationship with writing
}

María Concepción Sánchez Ramos
conchimary65@yahoo.es
https://orcid.org/0000-0002-7707-8848

Universidad de Carabobo, Valencia, Venezuela.

Recibido: $16 / 08 / 21$

Aceptado: 10/11/21

\section{Resumen}

El propósito de este artículo es presentar algunos argumentos y discernimientos acerca de las Tecnologías de la Información y Comunicación (TIC), y su relación con la escritura para la construcción activa de nuevos conocimientos, las cuales sirven de apoyo para formar productores de textos competentes, preparados para afrontar los retos y los constantes cambios que impone la sociedad actual. El desarrollo de las TIC ha influido de forma innegable en nuestra forma de vivir, y, por tanto, como factor social ha impactado de alguna manera los paradigmas educativos, debido a que pueden llegar a ser un excelente recurso didáctico en el proceso de enseñanza aprendizaje. La metodología empleada es de carácter documental bibliográfico.

Palabras clave: tecnología de la información, escritura, conocimientos.

\section{Abstract}

The purpose of this article is to present some arguments and insights about Information and Communication Technologies (ICT), and their relationship with writing for the active construction of new knowledge, which serve as support to train competent producers of texts, prepared to face the challenges and constant changes imposed by today's society. The development of ICT has undeniably influenced our way of life, and therefore, as a social factor, it has impacted in some way the educational paradigms, because they can become an excellent didactic resource in the teaching-learning process. The methodology used is of a bibliographic documentary nature

Keywords: information technology, writing, knowledge. 


\section{Introducción}

La escritura es considerada como una actividad comunicativa importante en todos los ámbitos de la vida del hombre. Es considerada un proceso cognitivo de alta complejidad; autorregulado que requiere no sólo conocimientos conceptuales sino aptitudes, habilidades y actitudes para construir ideas, opiniones, con el fin de ser expresados adecuadamente a la audiencia a quien va dirigida (Cassany, 1993; Díaz y Hernández, 2012). Además, la escritura posee una naturaleza procesual que es recursiva, pues permite al escritor planificar, textualizar y revisar constantemente.

Ahora bien, en su enseñanza deberían considerarse ciertos objetivos que tienen que ver con el desarrollo de la metacognición del estudiante o la capacidad de tomar consciencia de los procesos y subprocesos necesarios para la producción escrita eficaz, de la autorregulación de las estrategias que podrían usarse para el alcance de los propósitos propuestos por parte del escritor. Esta supone la puesta en marcha de un conjunto de procesos cognitivos complejos organizados entre sí de un modo recurrente e influido a su vez por diferentes variables y factores modulares tales como el conocimiento del género o de la tarea, la capacidad de la memoria operativa.

Las tecnologías de información y comunicación, en adelante TIC, en la educación, según los expertos, constituyen un fenómeno de gran trascendencia social, son un medio para potenciar la educación a partir del cual se puede fortalecer el proceso de la lectura y la escritura, dado que los estudiantes son hoy más sensibles a un entorno digital, porque posibilita un mayor grado de interacción con dispositivos electrónicos, teléfonos móviles, televisión digital, videojuegos y el uso habitual del internet. Dependiendo de la forma como se implementen, las TIC pueden tener un impacto que facilite o restrinja su uso. Esto lleva a reflexionar sobre la forma como se deben aprovechar las TIC, para que éstas sean implementadas en favor de la comunidad y no en su contra.

Así pues, la comunicación ha tenido un papel fundamental desde el principio de la creación del hombre, permitiendo interactuar con las personas y con el medio que lo rodea. El auge de las TIC, ha permitido que este proceso de interacción humana sea más rápido, ágil, y potenciado, facilitando la interconexión entre personas e instituciones educativas, eliminando (o al menos disminuyendo) las barreras espaciales y temporales que limitan el encuentro exclusivamente presencial.

En el contexto educativo, las TIC se reconocen como un conjunto de herramientas que permiten desarrollar una acción educativa que puede ir más allá de enfoques tradicionales; se está desplazando del aula de clase hacia la educación en línea o en la red. La educación virtual, por ende, deja a un lado y a su vez rompe con el paradigma de los esquemas de la educación clásica, memorística y tradicional, se generan no solo cambios en la creación de nuevos conocimientos, sino también en la forma de adquirir y repensar dichos saberes. 
La educación desempeña un papel muy importante en el desarrollo integral del ser humano y las TIC a lo largo de los tiempos, ha ido posicionándose de forma relevante dentro del proceso de enseñanza y aprendizaje, hasta lograr un sitial especial en la configuración del proceso educativo, permitiéndole a sus protagonistas obtener herramientas y aprovecharlas para potenciar su capacidad de enfrentar las situaciones académicas propuestas y atender a los retos planteados en la sociedad actual.

Los grandes cambios científicos y tecnológicos gestados han impuesto una nueva realidad a la escuela y más aún al docente de hoy han replanteado las posturas ortodoxas de la educación, las cuales se caracterizaban por desarrollar una comunicación vertical dentro de una dinámica formativa donde el docente era el conocedor absoluto del saber en la materia que impartía, mientras que sus alumnos se convertían en receptores mecánicos y modeladores de conductas instruidas. Las tendencias actuales, apuntan hacia un proceso formativo abierto, multidireccional, horizontal, reflexivo, crítico y emancipador, basado en el diálogo intersubjetivo.

Asimismo, se hace énfasis en el acompañamiento guiado para orientar al alumno en el descubrimiento de sus conocimientos, a través del aprovechamiento de sus estilos y ritmos de aprendizaje en la construcción de saberes. Junto a estos cambios, también se han ideado nuevas metodologías formativas que se apoyan en las TIC, las cuales son herramientas esenciales de trabajo y aprendizaje en la sociedad actual donde la generación, procesamiento y transmisión de información conforman un factor esencial de poder y productividad, en consecuencia, resulta cada vez más necesario educar para la sociedad de la información desde las etapas más tempranas de la vida escolar y ofrecen un abanico de posibilidades; tal es el caso de la educación B-Learning concebida como una modalidad educativa de formación profesional que trasciende las limitaciones de tiempo y espacio, donde se combina la utilización de metodologías didácticas y dialógicas mediadas por la interacción con las herramientas tecnológicas, para la construcción del conocimiento y de experiencias significativas.

En la era globalizada que se está viviendo, los avances científicos y tecnológicos están jugando un papel de suma importancia en la manera que los estudiantes se acercan tanto a la escritura como a la lectura. De lo anterior, se desprende la intención del presente escrito, en reflexionar en torno a la importancia de la TIC y su relación con la escritura para la construcción activa de conocimientos.

\section{El uso de las (TIC) como un modelo de enseñanza}

Partiendo de las ideas expuestas en el apartado anterior, las TIC son herramientas esenciales de trabajo y aprendizaje en la sociedad actual donde la generación, procesamiento y transmisión de información es un factor esencial de poder y productividad. En consecuencia, resulta cada vez más necesario educar para la sociedad de la información desde las etapas más tempranas de la vida escolar. Las potencialidades que ofrecen las herramientas de las TIC permiten la participación activa del estudiante en la construcción de su conocimiento. Aprender de manera diferente

Las tecnologías de la información y su relación con la escritura. - Eduweb, 2021, septiembre-diciembre,
v.15, n.3./62-68 
impone replantear el modelo pedagógico a la luz de los cambios que se han producido en el paradigma actual de aprendizaje, que lo asume como un proceso de construcción de conocimientos basado en la experiencia del que aprende. Las TIC permiten recrear ambientes organizadores de aprendizajes complejos, estimular el trabajo colaborativo, examinar materiales en diversos modos de presentación y perspectivas diferentes, estimular la reflexión y la negociación.

Las TIC desde su surgimiento hace un par de décadas aproximadamente, han ido jugando un papel trascendental en las formas de concebir las estrategias pedagógicas, permitiendo a diversos investigadores rediseñar las formas de generar conocimiento y transmitirlo adecuadamente a los estudiantes, quienes adquieren más competencias, sumando un componente virtual a la enseñanza convencional y presencial. Como lo indica Vargas (2015), la educación se ha ido transformando en función a los recursos que la sociedad provee al devenir, considerando los movimientos surgentes y cómo se redimensionan las estrategias pedagógicas en función a las necesidades de aprendizaje presentes.

Cabe destacar que el procesador de textos permite que el escritor se concentre selectivamente en cada una de las frases de la composición: generar ideas, organizarlas, redactar. Así como también, permite al escritor librarse de los aspectos más mecánicos de la composición escrita como lo son, la ortografía, puntuación, gramática, permitiendo así concentrarse absolutamente en la elaboración del contenido de texto.

Pues bien, las TIC, son un medio maravilloso para expresar las ideas y los pensamientos. La realidad de hoy es que se evidencia que muchos docentes se niegan hacer uso de todas las preeminencias que ofrecen las distintas plataformas tecnológicas creadas con fines pedagógicos, el reto debería ser que los estudiantes escribieran más utilizando las TIC. Sandia, Luzardo y Aguilar (2016) señalan que la "incorporación y apropiación de las TIC implica un cambio radical en la visión que se tiene del proceso educativo" (p. 106). Este propósito debería ser el motor esencial para impulsar el acto lector y de esa manera generar ideas que luego puedan fijarse en un papel o en cualquier soporte digital, y así aumentar la necesidad de organizar el pensamiento a través de la palabra escrita.

\section{Ventajas y desventajas de las TIC}

Como ya se ha mencionado anteriormente, las TIC son herramientas que permiten facilitar el trabajo docente. Sin embargo, hay que considerar que no reemplaza su rol de mediador y guía de los estudiantes. Por ello, es sustancial que se conozcan las ventajas que se pueden obtener al utilizar correctamente los medios tecnológicos, como también las desventajas que se pueden presentar al hacer uso de manera inapropiada. A continuación, se presentan algunas ventajas y desventajas de las TIC en la educación según Cacheiro (2014) 
Tabla 1.

Ventajas y desventajas de las TIC.

\begin{tabular}{|c|c|}
\hline Ventajas & Desventajas \\
\hline $\begin{array}{l}\text { *osee un alto poder de motivación, } \\
\text { aumentando el interés y la atención por las } \\
\text { tareas, siempre y cuando se utilicen } \\
\text { adecuadamente. }\end{array}$ & $\begin{array}{l}\text { * Puede suponer distracción siempre y } \\
\text { cuando no se tenga la correcta } \\
\text { planificación del proceso de enseñanza- } \\
\text { aprendizaje. }\end{array}$ \\
\hline $\begin{array}{l}\text { * Apoyo a los estudiantes con necesidades } \\
\text { educativas, a través de recursos y medios } \\
\text { adaptados que favorecen la comunicación } \\
\text { y a elaboración de tareas }\end{array}$ & $\begin{array}{l}\text { * No toda la información existente en el } \\
\text { internet es adecuada y para ello se debe } \\
\text { reconocer su tipo de procedencia y } \\
\text { alcance. }\end{array}$ \\
\hline $\begin{array}{l}\text { * Optimiza la capacidad de expresión del } \\
\text { estudiante, puesto que permite la } \\
\text { reelaboración de documentos de manera } \\
\text { más rápida y sencilla, utilizando } \\
\text { proaramas que avudan a las correcciones. }\end{array}$ & $\begin{array}{l}\text { * Las TIC no enseñan o preparan las } \\
\text { clases por sí solas, por lo que se requiere } \\
\text { de una formación por parte del discente y } \\
\text { del docente para que el potencial del uso } \\
\text { de las mismas sea el más alto posible. }\end{array}$ \\
\hline
\end{tabular}
Sánchez (2019)

\section{Escritura para construir nuevos conocimientos}

Estudios realizados en las últimas décadas, basados en la psicolingüística y en la psicología cognitiva, han permitido explorar la escritura como un proceso de construcción de significados que requiere de un complejo trabajo cognitivo. Se trata de un proceso que demanda la progresiva construcción de esquemas conceptuales que permitan interpretar datos previos y nuevos datos para transformarlos en conocimiento.

El proceso de escritura hoy es descrito como una actividad cognitiva compleja, debido a que el escritor, durante esta tarea pone en funcionamiento una serie de estrategias y conocimientos, es decir, un conjunto de operaciones que interactúan entre sí para llegar a la construcción del texto. Para que la escritura pueda desenvolverse de manera productiva requiere de subprocesos, que en íntima relación, suceden simultáneamente, de modo recursivo en la escritura: la planificación o preparación, la textualización o elaboración de borradores, la revisión y la edición definitiva (Cassany et. Al, 1993). Procesos que no se dan siempre obligatoriamente al escribir, puesto que depende de las circunstancias de producción, del tema, de los propósitos y de la audiencia.

De acuerdo con la psicolingüística y en el marco de la concepción constructivista del aprendizaje, se ha convertido en una herramienta de aprendizaje y en un saber que puede ser aprendido y consiguientemente enseñado. Esto significa entonces que en la escuela, la escritura debe ser concebida y utilizada de la misma forma que en la vida social: como un instrumento necesario para acceder al mundo del saber, para expresar puntos de vista, para comunicar sentimientos, emociones, experiencias, reflexiones, para informar a otros y para dejar constancia en el tiempo de saberes descubiertos. Por lo tanto, las situaciones de aprendizaje de la escritura en la escuela deben tener una

66 Las tecnologías de la información y su relación con la escritura. - Eduweb, 2021, septiembre-diciembre,
v.15, n.3./62-68 
intención comunicativa y un contexto social real, para que los estudiantes la perciban como una actividad significativa y necesaria para su formación.

\section{La composición escrita}

Investigaciones realizadas acerca de la composición escrita, hacen una distinción entre la escritura como proceso y la escritura como producto. Al respecto, existen diferentes concepciones necesarias de conocer para ampliar aspectos inherentes a este estudio. A continuación, se ofrece un breve recorrido teórico sobre estas concepciones.

Aunque la escritura aparece como un campo de estudio relativamente nuevo, éste se ha desarrollado de forma coherente. Desde la perspectiva del asociacionismo, según Ramos (2003), la composición era concebida como el influjo normativo y prescriptivo de la gramática. Escribir era un fenómeno mecánico centrado estrictamente en la representación gráfica de los datos lingüísticos proporcionados por fuentes externas. Sólo se prestaba importancia a las reglas ortográficas, los componentes teóricos, morfo lingüísticos y sintéticos vistos como un producto final.

Visto de esta manera, la composición escrita debe ser abordada desde un enfoque comunicativo y funcional y no como una simple repetición grafo motora de letras y signos. Mata y Arroyo (2005), sostiene que la coherencia en la investigación acerca de la composición escrita viene marcada de la concepción compartida por todos los investigadores de que la escritura es inseparable de los procesos lingüísticos, comunicativos y literarios. El movimiento intelectual sobre la escritura iniciado en los años cuarenta ha evolucionado desde la epistemología formalista centrada en el texto pasando por las teorías psicológicas centradas fundamentalmente en los procesos, hasta las teorías actuales más dialógicas que se centran tanto en el texto como en el proceso.

\section{El acto de escribir}

Según Cassany (1990), la escritura es una manifestación de la actividad lingüística humana. Escribir es un poderoso instrumento de reflexión. En el acto de escribir, los redactores aprenden sobre sí mismo y su mundo para comunicar las percepciones a otros. Escribir confiere el poder de creer como persona y de inferir en su mundo. El acto de escritura se materializa a través de un proceso en el cual el redactor imagina la audiencia, formula objetivos, desarrolla ideas, produce anotaciones, borradores y un texto elaborado que corrige para satisfacer las expectativas de la audiencia. A medida que el proceso se desarrolla, el redactor realiza cada una de estas tareas en cualquier momento. Se puede enseñar a los alumnos a escribir con más eficacia animándoles a aprovechar todo ese conjunto de actividades que comprende el acto de escritura. 


\section{A manera de conclusión}

La escritura es un proceso comunicativo continuo del ser humano, un desempeño de sus competencias comunicativas por medio de la palabra y ese desempeño está siempre en vías de ser mejorado y ampliado Cada uno de nosotros podemos encontrarnos con situaciones comunicativas nuevas en la cual se necesita producir o interpretar textos orales o escritos que nunca había producidos o interpretado antes.

Ahora bien, las TIC son herramientas pedagógicas que actualmente se consideran necesarias en cualquier área del aprendizaje, puesto que hacen parte de los contextos sociales y familiares que frecuentan a diario los estudiantes, además se relacionan estrechamente con la lectura y escritura. Es decir, estas fortalecen, enriquecen y complementan en gran nivel los procesos de lectura y escritura, dado que si los estudiantes hacen buen uso de estas pueden encontrar temas de su interés, lo que facilita la comprensión de nuevos tópicos.

Reflexionar en torno a la actividad de escritura permite la apertura de un camino a nuevas formas de problematizar los fenómenos relacionados con los seres humanos muy especialmente lo que en la actualidad se vive con respecto a la influencia de las TIC y los nuevos soportes digitales que están generando en las mente de los jóvenes y a su vez como, tal vez, sin proponérselo han desvalorizado actividades como la lectura y escritura que son la médula del conocimiento y del desarrollo cognitivo individual, siendo éstas en definitiva las protagonistas, ejecutoras y propulsoras del desarrollo de la sociedad del conocimiento.

La enorme potencialidad que las herramientas tecnológicas ponen hoy a nuestro alcance hace inevitable el pensar aprovecharlas como apoyo en el desarrollo de los procesos de enseñanza aprendizaje, debido a que representan un recurso de gran versatilidad que han revolucionado a la sociedad de forma general, transformando el tiempo y el espacio.

\section{Referencias}

Cacheiro, M. (2014). Educación y Tecnología: Estrategias Didácticas para la integración de las TIC. Madrid, Edición Digital. (e-pub) mayo de 2014.

Cassany, D. (1990). Enfoques didácticos para la enseñanza de la expresión escrita. Recuperado de: http://www.upf.edu/pdi/dtf/daniel_cassany/enfoques.htm

Cassany, D. (1993). La cocina de la escritura. Barcelona: Empúries.

Díaz, F y Hernández, G. (2012). Estrategias docentes para un aprendizaje significativo. México, Mc Grawn-Hill.

Mata y Arroyo. (2005). El proceso de planificación en la composición escrita de alumnos de la Educación Primaria. Revista de Educación. No 336, 353-376.

Ramos, J. (2003). Enseñar a escribir sin prisas...pero con sentido. Sevilla: M.C.E.P.

Sandia, B., Luzardo, M. y Aguilar, A. (2016). Una visión del nivel de apropiación de las TIC en la Universidad de Los Andes. Revista Educere. (035), 99-112.

Vargas, D. (2015). Las TIC en la educación. Plumilla Educativa, 62-79. Recuperado de: https://doi.org/10.30554/plumillaedu.16.1598.2015. 